\title{
Optical Losses in Hybrid Microcavity Based in Porous Semiconductors and its Application as Optic Chemical Sensor
}

\author{
Claudia Antonio Hernández ${ }^{1, b}$, Edith Osorio², Raúl Urteaga ${ }^{3}$, \\ Roberto Koropecki ${ }^{3}$, José Alberto Alvarado ${ }^{1}$ and Héctor Juárez ${ }^{1, \mathrm{a}^{*}}$ \\ ${ }^{1}$ Benemérita Universidad Autónoma de Puebla, CIDS-ICUAP, 14 Sur y Av. San Claudio, \\ s/n, San Manuel, 72570 Puebla, México. \\ ${ }^{2}$ CONACYT-Universidad de Quintana Roo, Boulevard Bahía s/n esq. Ignacio Comonfort, 77019 \\ Quintana Roo, México. \\ ${ }^{3}$ Instituto de Física del Litoral (CONICET-UNL), Güemes 3450,3000 Santa Fe, Argentina. \\ ajuarezsantiesteban@gmail.com, bclaudianhz@gmail.com
}

Keywords: Hybrid Structure, Transmittance spectrum, Porous Silicon, Optic Sensor, Nanoporous Anodic Alumina.

\begin{abstract}
In this study the experimental and theoretical optical analysis of a hybrid microcavity $(H M)$ based in porous silicon $(P S)$ and nanoporous anodic alumina $(N A A)$ are presented. The microcavity was centered in the visible region at $760 \mathrm{~nm}$. Distributed Bragg reflector (DBR) was obtained using galvanostatic anodizing method and while NAA by the two-step anodization technique. From SEM micrographs the HM different regions are observed. HM optical characterization in the visible region was done, considering two different light sources, point and non-point respectively. These results reveal a decrease in the quality factor $(Q)$ from 350 to 190 when the source is exchanged; this behavior has been mainly attributed to the light scattering at NAA. Furthermore, it was possible to study Q change, through transmittance simulation using the transfer matrix and Landau-Lifshitz-Looyenga theoretical methods. When a point light source is used, there are no optical losses making possible to sense $1 \%$ of analyte resulting in a $0.29 \mathrm{~nm}$ redshift of the resonant peak. According with these results we propose to apply the HM as chemical optic sensor.
\end{abstract}

\section{Introduction}

Optical devices based on porous materials have gained great interest due to their fast time response and their structural parameters that can be easily controllable. A simple technique such as electrochemical anodization has been commonly used to obtain these kinds of materials due to low cost fabrication and easy handle. Some materials obtained by this method are porous silicon (PS) and nanoporous anodic alumina $(N A A)$, both have been applied as optical waveguides [1], optic gas sensors [2-5], biosensors [6,7] and also in the medical area [8,9]. Besides, when they are exposed to analytes (vapors or immersed in organic solvents), a small change occurs on its optical properties, making them suitable to be applied as sensors.

PS multilayer structures have been proposed as chemical sensors due to its high surface area when the morphology changes [3-6]. The morphology can be reached under special synthesis conditions during the PS anodization process; where an specific in-depth porosities profiles can be obtained when a controlled current density is applied [4,10]. This process is self-limited which is an advantage, in other words the porous profile is only affected when the current density is modified during the fabrication process without altering the already made PS structure. However, the main disadvantage of porous silicon is the high absorption coefficient in the visible region and when is applied as Distributed Bragg Reflectors $(D B R)$ and microcavities, results in a poor optical quality value [2,11-13]. On the other hand, NAA presents good transparency from ultraviolet to midinfrared range, furthermore, by its physical and chemical properties (i.e. biocompatibility, thermal stability, environmental resistance, biodegradability, well-controlled geometry, etc.), makes it an excellent material to develop optical biosensors [7,14,15]. Nevertheless, this material also possesses 
a limiting factor, which is a lower refractive index values, making its applications as DBR complex [16-18].

Currently many research groups have reported optical sensors based on PS microcavities (using point light source); with high sensitivity at the infrared region, this structures possess a very narrow resonance peaks, high transmission and high quality factor $(Q)(1400-3300)[19,20]$, however NAA Q values (25-110) are smaller compared to PS ones[21,8].

In this work we exploited the optical properties of each material: PS refractive index and NAA low absorption, to develop a hybrid microcavity $(H M)$ which operates on the visible range $(760 \mathrm{~nm})$. Q values obtained in this work are better than those reported in the literature for structures based in PS, to reach this enhancement in our results a point light source was considered.

Several simulations were carried out to obtain the HM optical response when the structure is filled with different alcohol concentrations using non-point light source. These results reveal that the light scattering is due to the different domain contribution, noticing that all the optical losses occur mainly at the NAA membrane.

\section{Theoretical Aspects}

Transfer matrix method was used for HM theoretical analysis. This method describes the light transmission behavior through an isotropic homogeneous and inhomogeneous medium [22]. However, is well known that porous alumina is composed by different domains, the difference in morphology results in to an anisotropic and inhomogeneous hybrid structure [23,24].

Homogeneous and inhomogeneous structures Transmittance spectra was calculated taking into consideration different contributions such as: a whole HM gradient-optical thickness (GOT), incident angles $(\theta)$ and the wavelength range $(\lambda)$.

First simulation: transmittance calculus was done using the transfer matrix considering a point light source and an isotropic homogeneous medium (eq. 1):

$$
\left[\mathrm{M}_{\mathrm{i}}\right]=\left[\begin{array}{cc}
\cos \delta_{\mathrm{i}} & -\frac{\mathrm{i} \sin \delta_{\mathrm{i}}}{\gamma_{\mathrm{i}}} \\
-\mathrm{i} \gamma_{\mathrm{i}} \sin \delta_{\mathrm{i}} & \cos \delta_{\mathrm{i}}
\end{array}\right]=\left[\begin{array}{ll}
\mathrm{m}_{11}^{\mathrm{i}} & \mathrm{m}_{12}^{\mathrm{i}} \\
\mathrm{m}_{21}^{\mathrm{i}} & \mathrm{m}_{22}^{\mathrm{i}}
\end{array}\right]
$$

Where the term $\delta_{i}=\frac{2 \pi}{\lambda} n_{i} d_{i}, \gamma_{i}=n_{i} \cos \alpha_{i}$ wich depends on theese parameters: $n_{i}$ refractive index, $d_{i}$ physical thickness and $\alpha_{i}$ absorption coefficients for a given i-layer.

HM Transmittance was calculated by the following expression;

$$
\mathrm{T}=\left|\frac{2 \gamma_{0}}{\gamma_{0} \mathrm{~m}_{11}+\gamma_{0} \gamma_{\mathrm{s}} \mathrm{m}_{12}+\mathrm{m}_{21}+\gamma_{\mathrm{s}} \mathrm{m}_{22}}\right|^{2}
$$

Where, $\gamma_{0}$ and $\gamma_{\mathrm{s}}$ are defined as the incident medium and the substrate physical parameters respectively.

Second simulation: a non-point light source and anisotropic inhomogeneous medium are considered in equation 3, which can be described as the total sum of contributions from each domain presented at HM surface:

$$
\mathrm{T}_{\mathrm{t}}(\lambda)=\sum_{\theta_{\mathrm{i}}}^{\theta_{\mathrm{f}}} \sum_{\mathrm{GOT}_{\mathrm{i}}}^{\mathrm{GOT}_{\mathrm{f}}} \mathrm{T}\left(\theta_{\mathrm{i}}, \mathrm{GOT}_{\mathrm{i}}\right)
$$

When an amount of analyte is adsorbed on the inner part of the porous structure, the porous material effective medium is modified; affecting the refractive index. To calculate the complex refractive index $\left(\mathrm{n}_{\text {eff }}=\sqrt{\varepsilon_{\text {eff }}}\right)$ of each layer, Landau-Lifshitz-Looyenga $(L L L)$ model $[25,26]$ was used (eq. 4): 


$$
\varepsilon_{\mathrm{eff}}^{(1 / 3)}=\mathrm{p} \varepsilon_{1}^{(1 / 3)}+(1-\mathrm{p}) \varepsilon_{2}^{(1 / 3)}
$$

Where, $\varepsilon_{e f f}$ is defined as the complex dielectric function, $(p)$ porosity, $\left(\varepsilon_{1}\right)$ air or analyte complex dielectric function, $\left(\varepsilon_{2}\right)$ silicon crystalline at PS or aluminum at NAA complex dielectric function, besides the HM simulation when it is filled with alcohol was done by Lorentz-Lorentz model.

\section{Experimental Details}

Distributed Bragg Reflector Fabrication. PS was prepared using galvanostatic anodization method where p-type (100) monocrystalline silicon wafers (resistivity $0.01-0.02 \Omega \mathrm{cm}$ ) were used as substrate. To prepare the desired solution for electrochemical etching, $\mathrm{HF}(50 \%): \mathrm{C}_{2} \mathrm{H}_{5} \mathrm{OH}$ $(99.9 \%)$ in a relation 1:2 (v/v) without any further purification, were mixed. Electrochemical cell is made by Teflon ${ }^{\circledR}$ beaker, where the $\mathrm{Si}$ wafer acts as anode and platinum wire as cathode [3]. The electrolyte contact area over the silicon substrate was $0.78 \mathrm{~cm}^{2}$. DBR's were designed to work at a specific wavelength (stop band, $\lambda \mathrm{c}=760 \mathrm{~nm}$ ) by alternating layers of higher $\left(P_{H}\right)$ and lower $\left(P_{L}\right)$ porosities, as follows; $\mathrm{P}_{\mathrm{L}}-\mathrm{P}_{\mathrm{H}}-\mathrm{P}_{\mathrm{L}}-\mathrm{P}_{\mathrm{H}}-\mathrm{P}_{\mathrm{L}}-\mathrm{P}_{\mathrm{H}}-\mathrm{P}_{\mathrm{L}}-\mathrm{P}_{\mathrm{H}}-\mathrm{P}_{\mathrm{L}}-\mathrm{P}_{\mathrm{H}}-\mathrm{P}_{\mathrm{L}}-\mathrm{P}_{\mathrm{H}}-\mathrm{P}_{\mathrm{L}}$. Current densities $\mathrm{J}_{\mathrm{L}}=5 \mathrm{~mA} / \mathrm{cm}^{2}$ and $\mathrm{J}_{\mathrm{H}}=80 \mathrm{~mA} / \mathrm{cm} 2$ were applied respectively, with an specific time of $\mathrm{t}_{\mathrm{L}}=24 \mathrm{~s}$ and $\mathrm{t}_{\mathrm{H}}=5 \mathrm{~s}$ to obtain physical thicknesses of $\mathrm{d}_{\mathrm{L}}=84 \mathrm{~nm}$ and $\mathrm{d}_{\mathrm{H}}=115 \mathrm{~nm}$ (according to the literature, the optical thickness of each layer needs to be at $\lambda / 4)$. Finally, PS-DBR refractive index $\left(n_{L}=2.24\right.$ and $\left.n_{H}=1.64\right)$ at $760 \mathrm{~nm}$ was obtained.

Nanoporous anodic alumina fabrication. NAA was obtained by two-step anodization process [27]; using high-purity (99.999\%) aluminum foils, $500 \mu \mathrm{m}$ thickness. Prior anodization step, Al substrates were chemically degreased by acetone, followed by native oxide removing using $0.5 \%$ $\mathrm{NaOH}$ solution, after the cleaning process all the samples were rinsed in deionized water and dried with $\mathrm{N}_{2}$. To obtain millimetric grain sizes and reduce NAA surface roughness, aluminum was annealed at $400{ }^{\circ} \mathrm{C}$ for $3 \mathrm{~h}$ and mechanically pressed $\left(7.5 \mathrm{ton} / \mathrm{cm}^{2}\right)$ at room temperature, after that a second annealing process with same parameters was done. This whole annealing and press process is known as dynamic recrystallization, which favors crystal growth from $\mu \mathrm{m}$ up to several millimeters $[28,29]$.

After thermal treatment, samples were electropolished with a stirred $\mathrm{HClO}_{4}: \mathrm{C}_{2} \mathrm{H}_{5} \mathrm{OH}(1: 4 \mathrm{v} / \mathrm{v})$ solution, under a constant voltage $(20 \mathrm{~V})$ for $10 \mathrm{~min}$ at room temperature. Next, first anodization step was carried out in $0.3 \mathrm{M} \mathrm{H}_{2} \mathrm{C}_{2} \mathrm{O}_{4}$ solution at $40 \mathrm{~V}$ for $20 \mathrm{~h}$, a Teflon cell with cooled system was used for anodization, maintaining the temperature constant at $7{ }^{\circ} \mathrm{C}$ under continuous stir, $\mathrm{Pt}$ wire and aluminum were used as a cathode and anode respectively. Subsequently, the alumina film was selectively dissolved by wet chemical etching in $0.4 \mathrm{M} \mathrm{H}_{3} \mathrm{PO}_{4}: 0.2 \mathrm{M} \mathrm{H}_{2} \mathrm{CrO}_{7}$ solution at $70{ }^{\circ} \mathrm{C}$ for $3 \mathrm{~h}$. Second anodization step was realized under the same chemical conditions as the first one, nevertheless in this step 4 hours anodization time was used the reach the physical thickness desired $(15 \mu \mathrm{m})$.

Hybrid Microcavity assemble. As it was mentioned before NAA and DBR structures were obtained separately, so an extra process is required to assemble the HM structure. First is necessary freestanding the DBR from the c-Si with $\mathrm{HF}(50 \%): \mathrm{C}_{2} \mathrm{H}_{5} \mathrm{OH}(1: 7 \mathrm{v} / \mathrm{v})$ chemical solution, applying $200 \mathrm{~mA} / \mathrm{cm}^{2}$ current density (4 s), after this, the DBR is rinsed with isopropyl alcohol under constant flux to assure no adherence over any other surface and free transferred over upper NAA surface, since the backside of NAA still attached over the aluminum substrate, is necessary to remove the substrate using $2.4 \mathrm{M} \mathrm{HCl}: 0.16 \mathrm{M} \mathrm{CuCl}_{2}$ saturated solution. After freestanding the other DBR, inmediately is deposited over the NAA remaining lone side, to have a total assemble of the HM as it is showed in Fig. 1. 

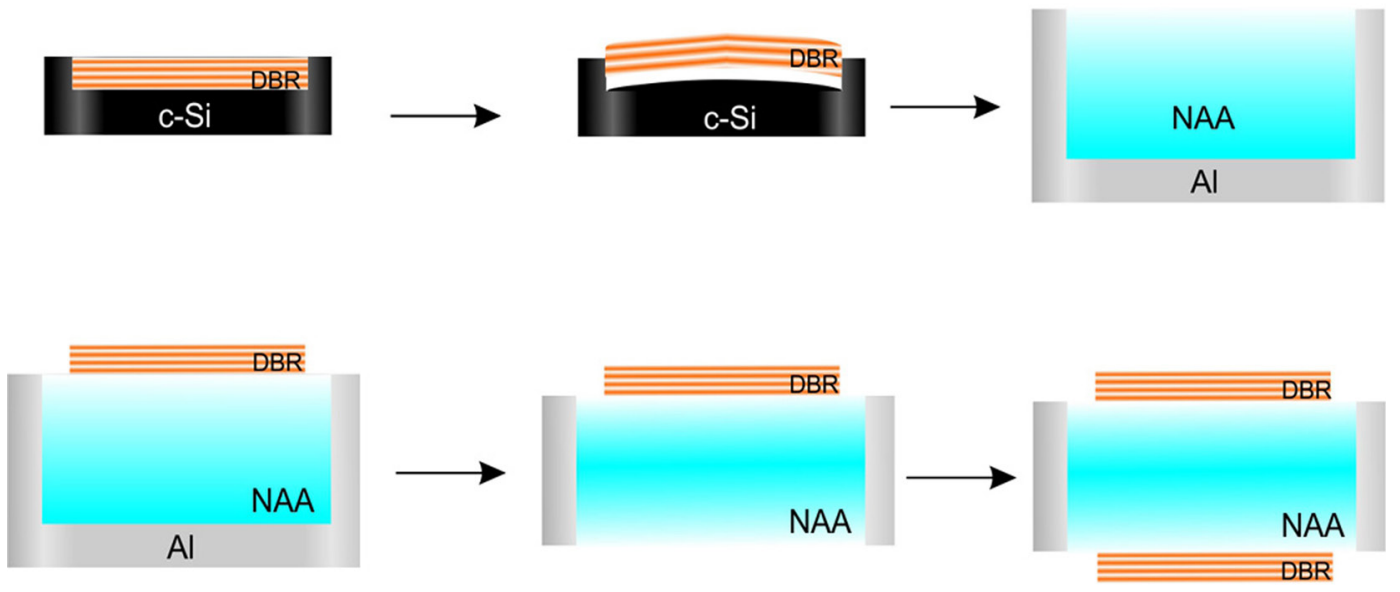

Fig. 1 Hybrid Microcavity assemble.

Characterization techniques. FEI Scios Dualbeam Scanning Electron Microscopy (SEM) was used to obtain cross-sectional and surface images from PS-DBR structures and NAA membrane. To observe the domains presented over NAA membrane, a CARL ZEISS metallographic microscope with circularly polarized light was used. The elemental composition of HM was obtained by Energy Dispersive X-Ray Analysis $(E D X)$.

Optical characterization was carrying out using a Evolution 600 UV-VIS spectrophotometer (non-point light source), the overall system wavelength resolution was $2 \mathrm{~nm}$. Point light source characterization was obtained using Ocean Optics HR400 spectrometer with $0.2 \mathrm{~nm}$ resolution.

HM optical transmittance spectra were carried out following two different setups. First setup: A non-point light source hits directly over several domains covering $9 \mathrm{~mm}^{2}$ total area, second setup: a point light source hits the sample on a very tiny area of $0.47 \mathrm{~mm}^{2}$, which corresponds to a single domain area, see figure 2 .

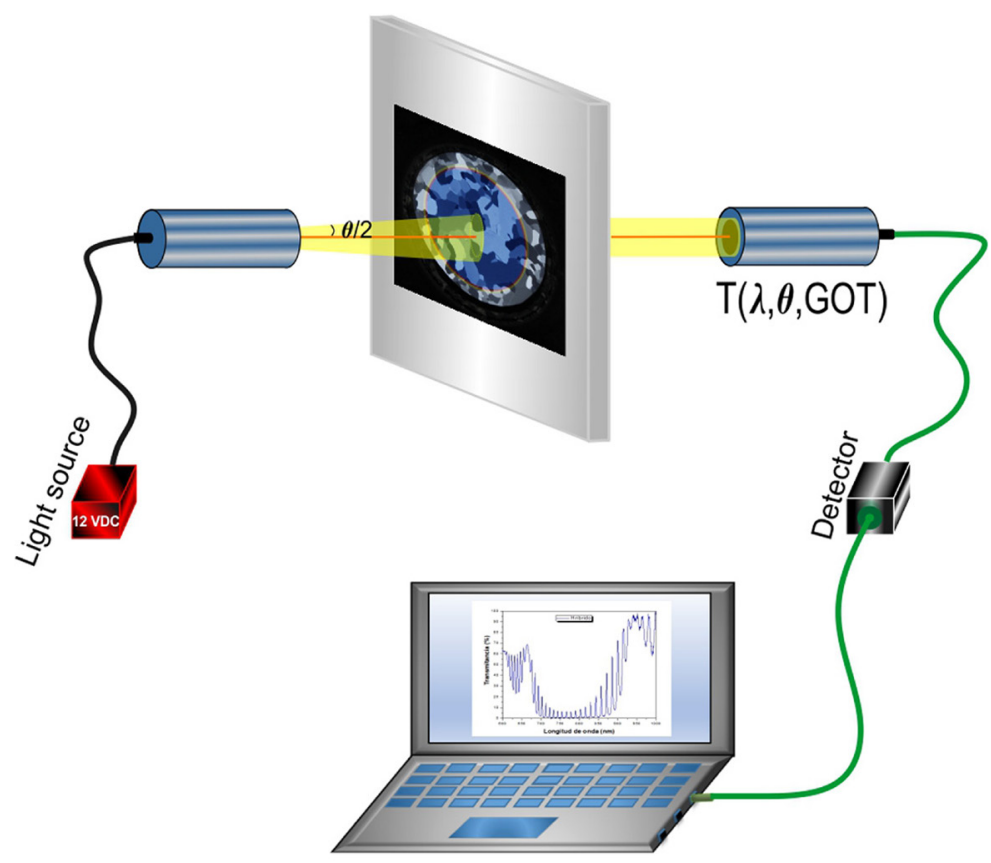

Fig. 2 Transmittance setup with a point (red line) and a non-point (yellow) light source.

\section{Results and Discussion}

Figure 3 shows the typical reflectance spectra (non-point light source) of a DBR centered at $760 \mathrm{~nm}$ normal incidence, the transfer matrix method and LLL's effective medium theory were 
used to fit experimental and theoretical data $[25,22,30]$. Optical parameters such as: refractive index and the physical thicknesses $\left(n_{L}=2.24, n_{H}=1.64, d_{L}=84 \mathrm{~nm}\right.$ and $\left.d_{H}=115 \mathrm{~nm}\right)$ for each layer were obtained.

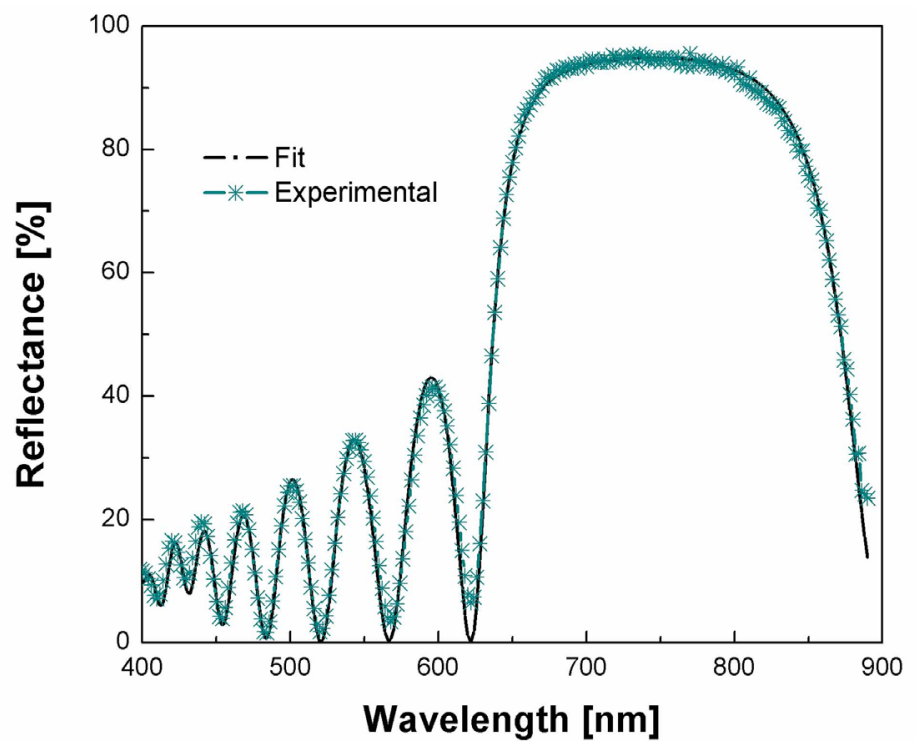

Fig. 3 PS-DBR typical reflectance spectra centered at $760 \mathrm{~nm}$ measured (black line) and fitted (cyan asterisks).

The optical micrograph (Fig. 4a) from NAA surface under polarized light is showed; several contrasts are observed corresponding to different domains due to the NAA birefringence. This effect is related to the different substrate crystalline orientations which contribute on the domains size (up to $1.9 \mathrm{~mm}$ in length) and shape [23]. Furthermore, each NAA domain contains pores with different degrees of ordering and tilts. For example, it has been reported for aluminum [001] crystalline orientation that NAA grows faster with high pore ordering compared to [101] plane [24], this characteristic favors the material anisotropy.

Figure $4 \mathrm{~b}$ NAA reflectance spectra over a single and many domains are shown. When point light source strikes a single domain the constructive interference amplitude is increased and well defined. Nevertheless, when the non-point light source strikes over several domains the constructive interferences tend to decrease and deformed due to different optical lengths in each domain, in other words there is an inhomogeneous surface that contributes to the reflectance optical losses.
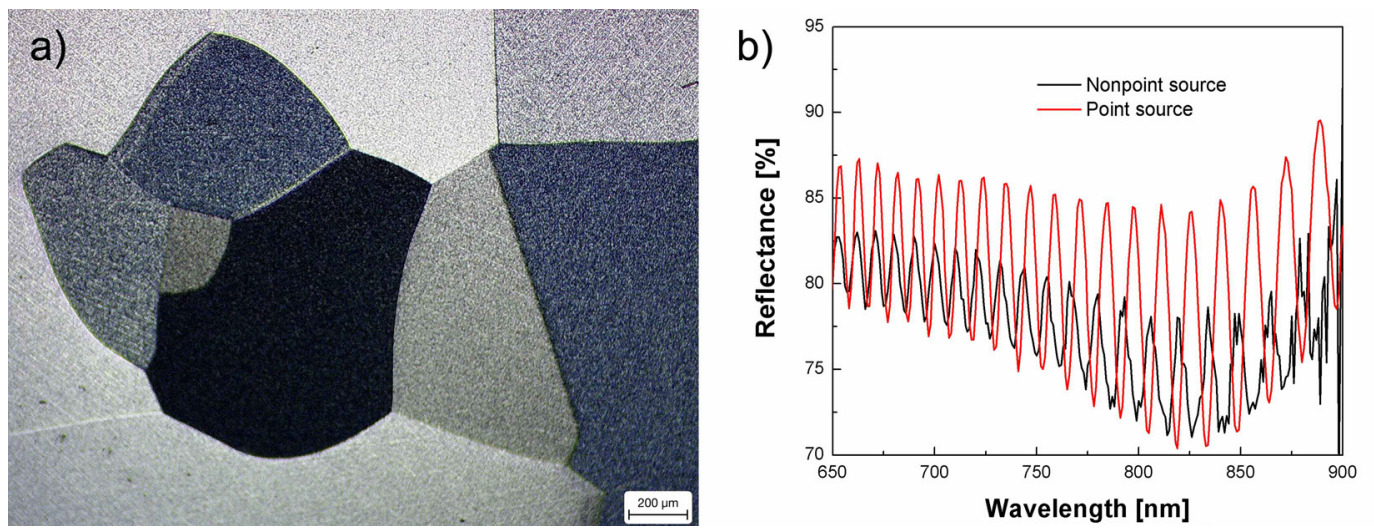

Fig. 4 NAA membrane characterization (a) different domains optical micrograph image under polarized light, (b) experimental reflectance spectra at normal incidence using an a point light source with $0.47 \mathrm{~nm}^{2}$ area (red line) and nonpoint source with $9 \mathrm{~mm}^{2}$ (black line).

In figure 5, the top-view PS and NAA SEM imagine are shown, the figure 5a) shows the PS SEM image where a random pore distribution and $11 \mathrm{~nm}$ average diameter are observed and figure $5 \mathrm{~b}$ ) corresponds to NAA, from this image a self-ordered pore is noticed, with $80 \mathrm{~nm}$ average diameter 
and $103 \mathrm{~nm}$ interpore distance. Furthermore, a quasi-periodic array is observed due to point defects that occur at the porous domain during the anodization process [31].
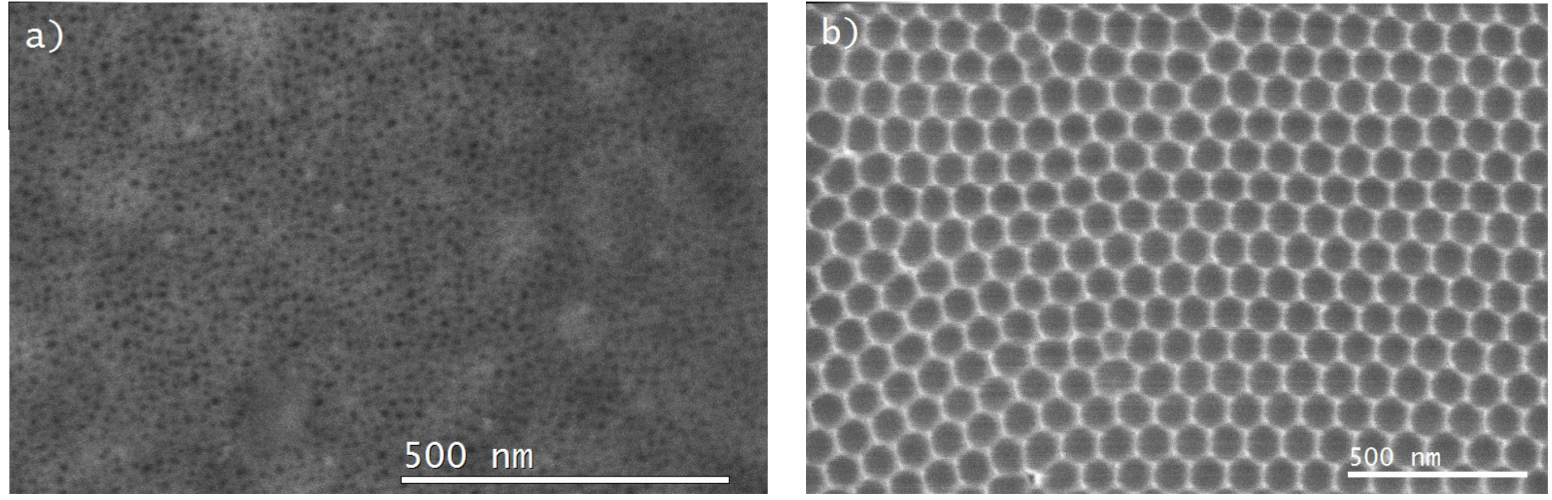

Fig. 5 SEM top images (a) porous silicon random pore distribution and (b) NAA resulting image with self-ordered pores.

DBR's were manufactured alternating low and high refractive index as mentioned above; refractive indexes used were 2.24 and 1.64 respectively, both DBR's were assembled to the NAA surfaces as it was mention before. Figure 6a) shows the cross-section SEM image from the hybrid microcavity, DBR / NAA / DBR different regions are clearly observed. Both DBR's have $1.6 \mu \mathrm{m}$ and NAA $18 \mu \mathrm{m}$ of physical thickness with cylindrical pores straight to the surface. Fig. $6 \mathrm{~b}$ refers to the elemental chemical composition obtained from Energy Dispersive X-ray Spectroscopy (EDX) analysis, these compositions belongs to the silicon from the PS and the aluminum-oxygen elements from the NAA respectively.

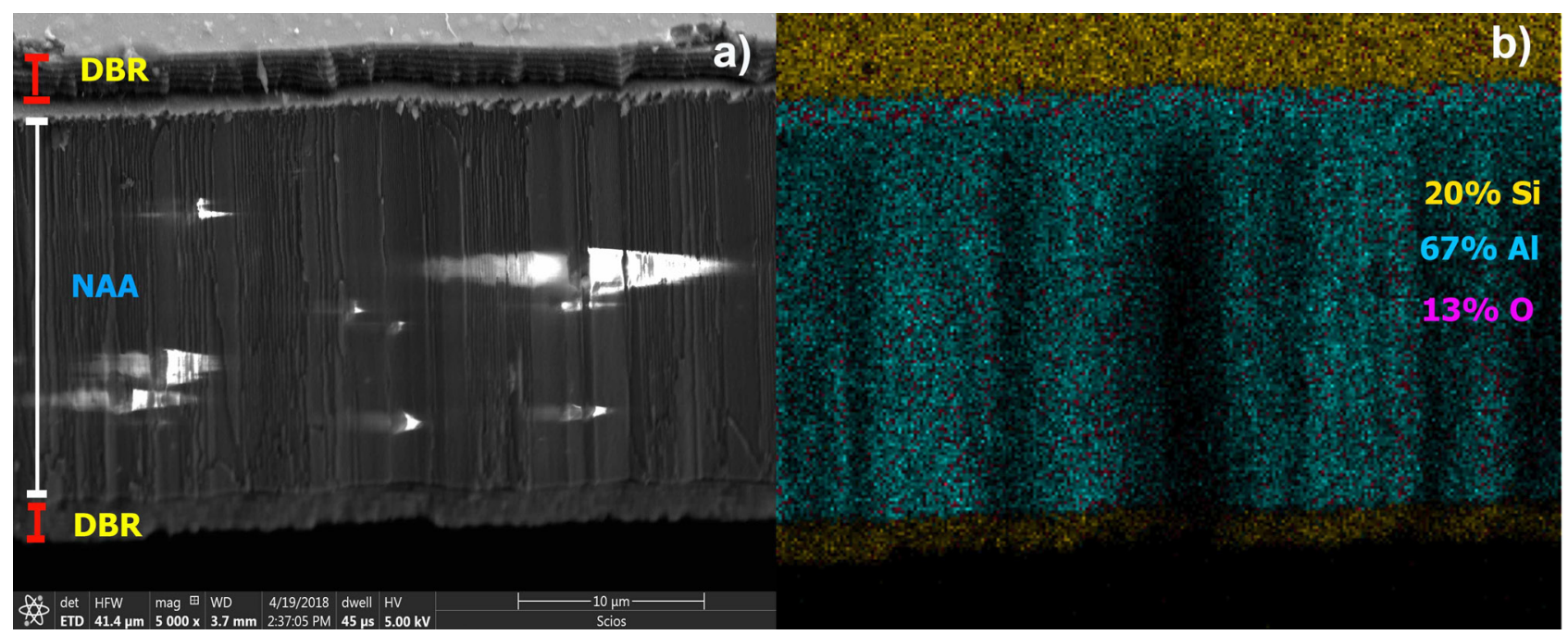

Fig. 6 Hybrid microcavity (a) cross-sectional SEM image and (b) elemental chemical composition of the final structure.

Transmittance spectra of the HM structure was carried out considering two different setups. The hybrid microcavity theoretical and experimental response was obtained under a point light source condition (Fig. 7); the transmittance spectra is fitted considering a HM homogenous surface (eq. 2), where the measured area was $0.47 \mathrm{~mm}^{2}$, considering a low absorption $\left(6 \mathrm{~cm}^{-1}\right)$, with $10 \%$ of porosity and $15 \mu \mathrm{m}$ of physical thickness.

With these conditions, the theoretical data was fitted to the experimental, resulting in a distributed uniform resonances contribution located at the stop band, where $Q$ factor value was 350 at $760 \mathrm{~nm}$. From these results we can observe that the resonance quantity is related directly to the NAA thickness. 


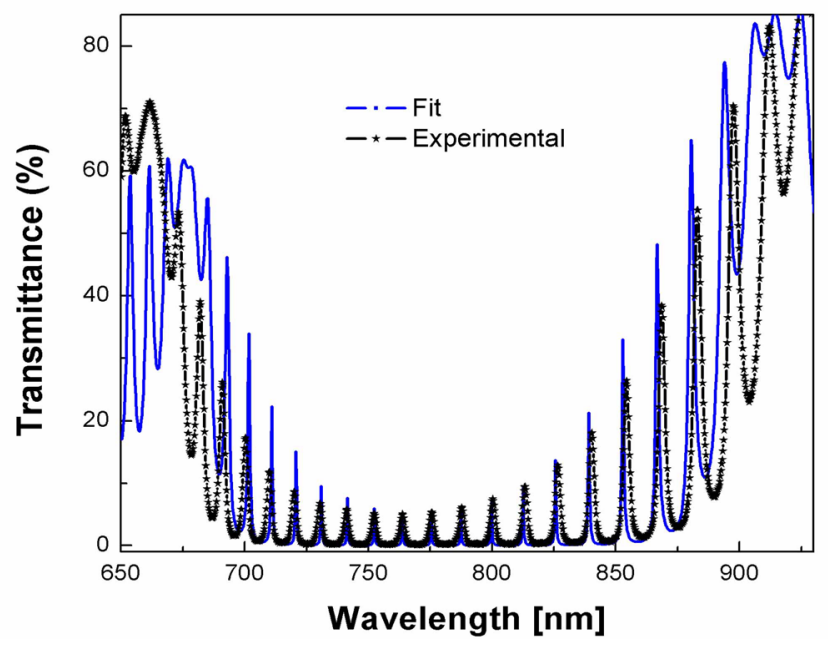

Fig. 7 Hybrid microcavity transmittance spectra experimental (black dots) and fitted (blue solid line).

Figure 8 shows the NAA transmittance spectra using a non-point light source where several domains contributions are considered, from the results it is observed that the amplitude decreases and a resonant peaks deformation is presented. The transmittance results were analyzed and fitted from theoretical to the experimental data (eq. 3) considering the porosity gradient and the material absorption (NAA domains). However, is known that the material absorption depends directly to the measured area and the light incident angle $\left(0^{\circ}-10^{\circ}\right)$. With these considerations, a theoretical fitting was carried out, a decrease in the transmittance intensity and Q values from 350 to 190 were observed, it could be due to the physical parameters related to the NAA surface. Furthermore, when different stop band $(760 \mathrm{~nm})$ values are considered, the theoretical fitting does not match with the experimental data due to optical losses caused by the optical thickness variations.

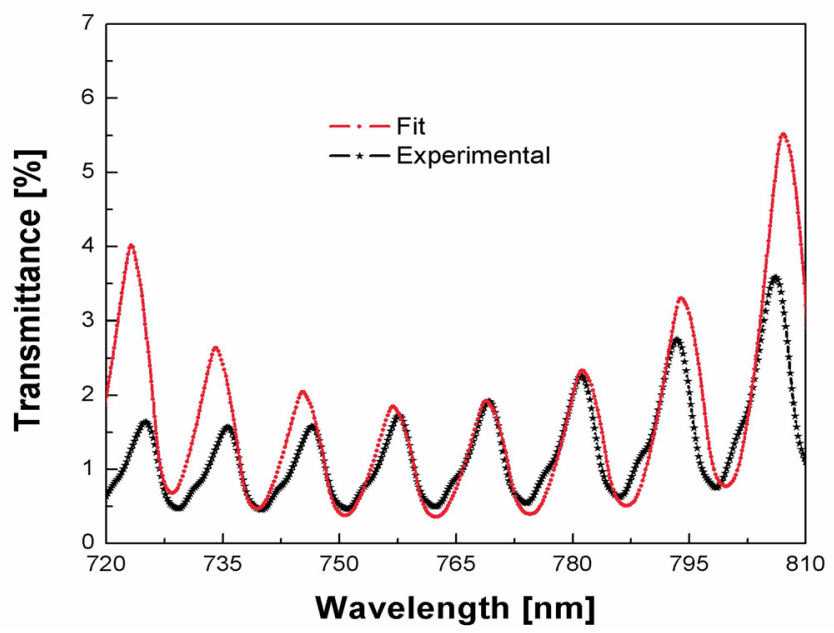

Fig. $8 \mathrm{HM}$ transmittance spectra considering a non-point light source, experimental (black dots) and simulated (red line).

To analyze the HM optical response several simulations were performed at different analyte concentrations. After multiple calculations, we calculated the maximum analyte volume $(0.2 \mu \mathrm{l})$ that can be introduced into the structure; this value was considered as the $100 \%$ and then used as reference volume for this research. To simulate the mixture of air and alcohol inside the pores, Lorentz-Lorentz model was used. Figure 9 shows the resonant peak simulation using a point light source where the structure full and empty of ethanol (blue dots and black dots ) is considered. When the analyte is getting inside the pores substituting the air, a redshift in transmittance spectra is presented due to the variation on the refractive index values. This behavior allows HM to be applied as an optical gas sensor. 


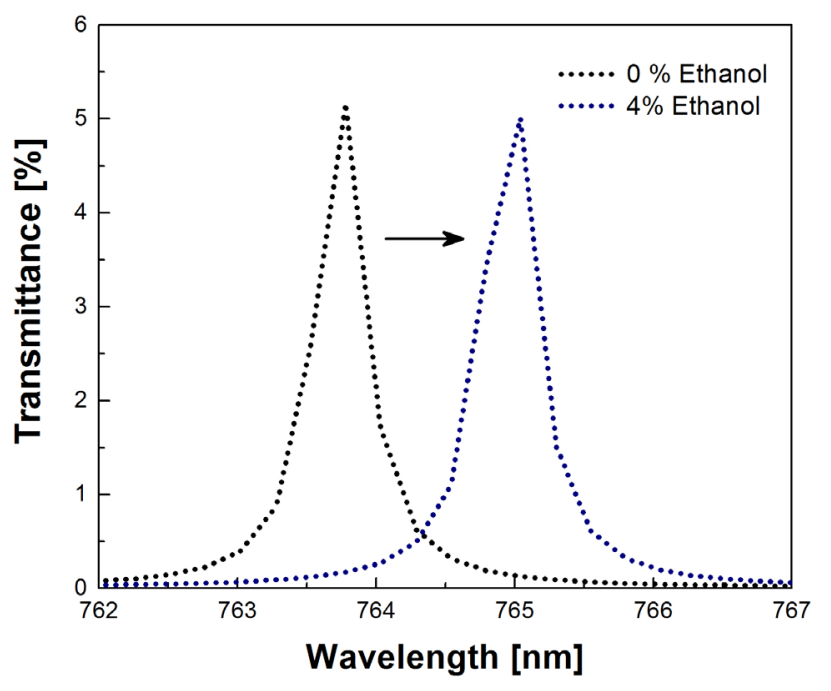

Fig. 9 Resonant peak redshift when pores are filled with $4 \%$ ethanol.

Figure 10 presents the evolution between alcohol concentration and the peak position when a point light source is used. For this analysis a peak located at $764 \mathrm{~nm}$ was used as an initial reference, and compared with those peaks when analyte percentage inside the structure is increased. Peak value tends to move towards longer wavelengths showing a linear behavior from $1 \%$ to $50 \%$ ethanol concentration (99.3\% fitted) (Figure 10a). The resonant peak shifts $0.29 \mathrm{~nm}$ proportionally whit the alcohol concentration (1\%) (Figure $10 \mathrm{a}$ inset). Theoretically $1 \%$ is the minimum analyte

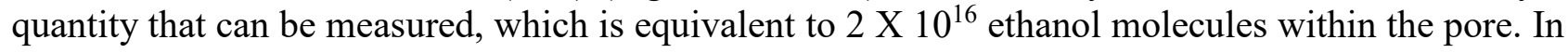
other words, it is possible to detect less than $1 \mathrm{ppm}$ of alcohol.

Using a non-point light source, same simulations were done in order to compare the HM optical response (Figure 10b). The results reveal that the resonant peak displacement only shows a short lineal behavior ( 5 - $30 \%$ alcohol concentration) but beyond this value a non-lineal tendency is observed. This effect happens when the domain optical properties contributes negatively provoking losses between the refractive indexes (PS and NAA) and only the analyte refractive index contribution is becoming meaningful. The minimum theoretical redshift that can be measured with this setup is $1.6 \mathrm{~nm}$ which corresponds to $5 \%$ analyte concentration.
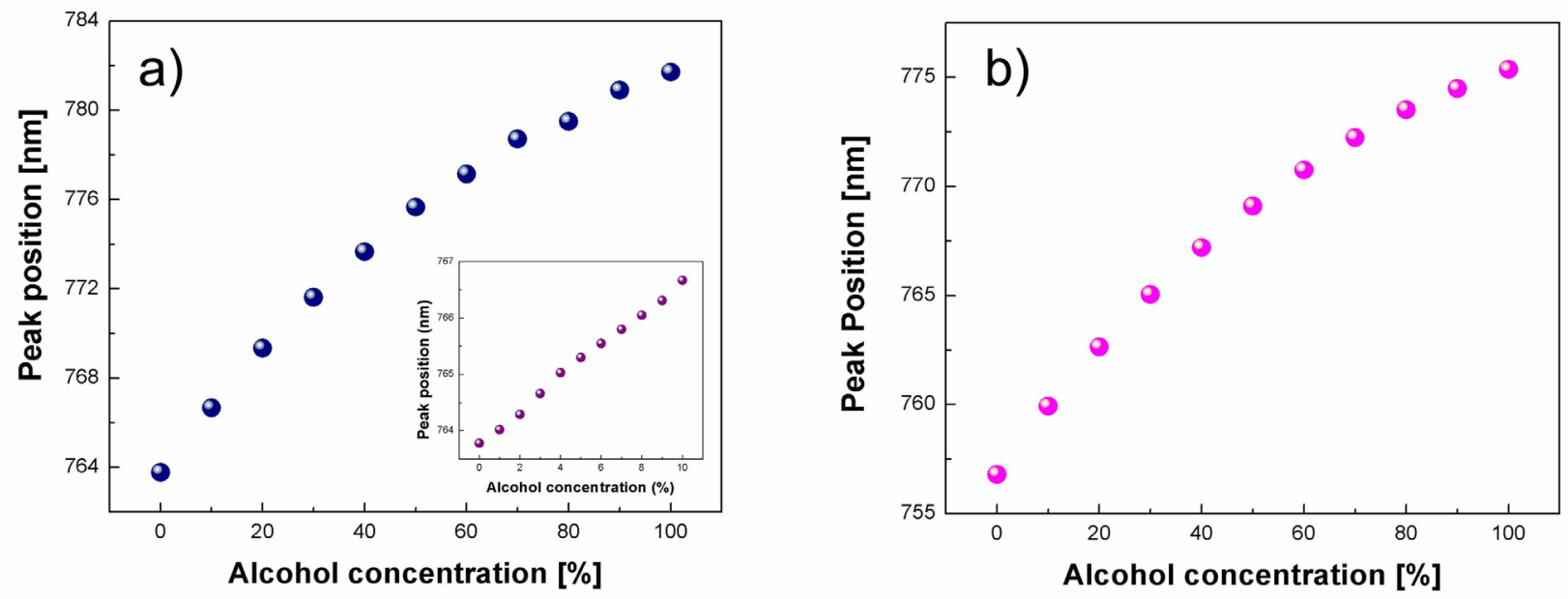

Fig. 10 Behaviour between peak position vs alcohol concentration (a) a point light source, (b) non-point light source. 


\section{Conclusions}

PS and NAA optical properties were used to obtain a microcavity with a $Q$ factor value of 350 and a physical thickness of $18 \mu \mathrm{m}$ that perfectly works in the visible region at $760 \mathrm{~nm}$. Experimentally two light sources (point and non-point) were used to obtain the transmittance, a light scattering on the HM optical response is observed when the light strikes over several NAA domains and a decrease on the Q value (350-190) is observed. LLL's model was used to fit the experimental data, showing that the light scattering is provoked by NAA non-uniformity. Different optical lengths were observed due to the domains presented on the structure. When an analyte is adsorbed a redshift occurs allowing to detect less than $1 \mathrm{ppm}$, with these results we conclude that is possible to apply the HM as chemical optic sensor.

\section{Acknowledgement}

Authors acknowledge the financial support from Consejo Nacional de Ciencia y Tecnología (CONACyT) in Mexico. We are grateful to Dr. Netzahualcóyotl Carlos and Dr. Mario Moreno for SEM analysis done at Instituto Nacional de Astrofísica, Óptica y Electrónica (INAOE).

\section{References}

[1] A. Loni, L. T. Canham, M. G. Berger, R. Arens-Fischer, H. Munder, H. Luth. H. Arrand, and T. M. Benson, "Porous silicon multilayer optical waveguides" Thin Solid Films 276, 143-146 (1996)

[2] V. Mulloni and L. Pavesi, "Porous silicon microcavities as optical chemical sensors", Appl. Phys. Lett. 76, 18, 2523 - 2525 (2000).

[3] E. Osorio, R. Urteaga, H. Juárez, and R.R. Koropecki, "Transmittance correlation of porous silicon multilayers used as a chemical sensor platform”, Sens. Actuators B. 213, 164-170 (2015).

[4] L.N. Acquaroli, R. Urteaga, and R.R. Koropecki, "Innovative design for optical porous silicon gas sensor", Sens. Actuators B. 149, 189-193 (2010).

[5] I. A. Levitsky, "Porous Silicon Structures as Optical Gas Sensors", Sensors 15, 19968-19991 (2015)

[6] F. A. Harraz, "Porous silicon chemical sensors and biosensors: A review", Sens. Actuators B. 202, 897-912 (2014)

[7] J. F. Borrull, J. Pallarès, G. Macías, L. F. Marsal, "Nanostructural Engineering of Nanoporous Anodic Alumina for Biosensing Applications", Materials 7, 5225-5253 (2014)

[8] Y. Wang, Y. Chen, T. Kumeria, F. Ding, A. Evdokiou, D. Losic, and A. Santos, "Facile Synthesis of Optical Microcavities by a Rationally Designed Anodization Approach: Tailoring Photonic Signals by Nanopore Structure", ACS Appl. Mater. Interfaces 7, 98799888 (2015)

[9] K. I. Hartmann, A. Nieto, E. C. Wu, W. R. Freeman, J. S. Kim, J. Chhablani, M. J. Sailor, and L. Cheng, J. Ocul. "Hydrosilylated Porous Silicon Particles Function as an Intravitreal Drug Delivery System for Daunorubicin”, Pharmacol. Ther. 29, 5, 493-500 (2013)

[10] O.Bisi, Stefano Ossicini and L.Pavesi, "Porous silicon: a quantum sponge structure for silicon based optoelectronics", Surf. Sci. Rep. 38, 1-126 (2000).

[11] M. H. Chan, S. K. So, and K. W. Cheah, "Optical absorption of free-standing porous silicon films”, J. Appl. Phys 79, 6, 3273-3275 (1996).

[12] V. Grivickas and P. Basmaji, "Optical absorption in porous silicon of high porosity", Thin Solid Films 235, 234-238 (1993) 
[13] L. Pavesi, "Porous silicon dielectric multilayers and microcavities", L. Riv. Nuovo Cim 20, $10,1-76(1997)$

[14] D. W. Thompson, P. G. Snyder, L. Castro, L. Yan and P. Kaipa, "Optical characterization of porous alumina from vacuum ultraviolet to midinfrared”. J. Appl. Phys. 97, 113511-1113511-9 (2005)

[15] A. Santos J. Mater. "Nanoporous anodic alumina photonic crystals: fundamentals, developments and perspectives", Chem. C 5, 5581-5599 (2017)

[16] C. S. Law, S. Y. Lim and A. Santos, "Fine tuning of transmission features in nanoporous anodic alumina distributed Bragg reflectors", Proc. SPIE 10456, Nanophotonics Australasia 2017

[17] J. G. J. Peelen and R. Metselaar, "Light scattering by pores in polycrystalline materials: Transmission properties of alumina", J. Appl. Phys 45, 216-220 (1974)

[18] R. Apetz and M. P. B. van Bruggen, "Transparent Alumina: A Light-Scattering Model”, J. Am. Ceram. Soc., 86, 480-486 (2003)

[19] P. J. Reece, G. Lérondel, W. H. Zheng, and M. Gal, "Optical microcavities with subnanometer linewidths based on porous silicon”, Appl. Phys. Lett. 81, 4895-4897 (2002)

[20] M. Ghulinyan, C. J. Oton, G. Bonetti, Z. Gaburro, and L. Pavesi, "Free-standing porous silicon single and multiple optical cavities”, J. Appl. Phys 93, 9724-9729 (2003)

[21] C. S. Law, S. Y. Lim, R. M. Macalincag, A. D. Abell, and A. Santos, "Light-Confining Nanoporous Anodic Alumina Microcavities by Apodized Stepwise Pulse Anodization", ACS Appl. Nano Mater 1, 9, 4418-4434 (2018)

[22] F. Pedrotti, Introduction to Optics (Prentice-Hall, Inc., 1993).

[23] L. Cencha, C. A. Hernández, L. Forzani, R. Urteaga And R.R. Koropecki, "Optical performance of hybrid porous silicon-porous alumina multilayers", J. Appl. Phys 123, 183101-1-183101-8 (2018)

[24] C. K. Y. Ng and A. H. W. Ngan, "Precise Control of Nanohoneycomb Ordering over Anodic Aluminum Oxide of Square Centimeter Areas", Chem. Mater 23 (23), 5264-5268 (2011)

[25] W.Theiß, “Optical properties of porous silicon”,Surf. Sci. Rep. 29, 3-4, 91-192, (1997)

[26] H. Sohn, "Refractive Index of Porous Silicon”, Handbook of Porous Silicon, 1-12 (2014)

[27] Masuda H and Fukuda K. "Ofdered Metal Nanohole Arrays Made by a Two-Step Replication of Honeycomb Structures of Anodic Alumina”, Science 268, 1466-1468 (1995)

[28] H. R. R. Ashtiani and P Karami, "Prediction of the Microstructural Variations of ColdWorked Pure Aluminum during Annealing Process", Modeling and Numerical Simulation of Material Science, 5, 1-14, (2015)

[29] D. F. Li, D. Z. Zhang, S. D. Liu, Z. J. Shan, X. M. Zhang, Q. Wang, and S. Q. Han, "Dynamic recrystallization behavior of 7085 aluminum alloy during hot deformation", Trans. Nonferrous Met. Soc. China 26, 6,1491-1497, (2016)

[30] M. J. Sailor, "Porous Silicon in Practice; Preparation, Characterization and Applications", Wiley-VCH, (C2012, pp.133-187

[31] F. Li, L. Zhang, and R. M. Metzger, "On the Growth of Highly Ordered Pores in Anodized Aluminum Oxide”, Chem. Mater., 10, 9,2470-2480 (1998) 